\title{
BRDF of Salt Pan Regolith Samples
}

\author{
Georgi T. Georgiev ${ }^{a}$, Charles K. Gatebe ${ }^{b}$, James J. Butler ${ }^{c}$, and Michael D. King ${ }^{d}$ \\ aScience Systems and Applications, Inc., Lanham, MD 20706, e-mail: georgi.t.georgiev@nasa.gov \\ ${ }^{b}$ Goddard Earth Science and Technology Center, University of Maryland Baltimore County, Baltimore, MD 21228 \\ "Earth-Sun Exploration Division, NASA Goddard Space Flight Center, Greenbelt, MD 20771 \\ ${ }^{d}$ Laboratory for Atmospheric and Space Physics, University of Colorado, Boulder, CO 80309
}

\begin{abstract}
Laboratory BRDF measurements of salt pan regolith samples are presented in this study in an effort to understand the role of spatial and spectral variability of the natural biome. The samples were obtained from Etosha Pan, Namibia $\left(19.20^{\circ} \mathrm{S}\right.$, $15.93^{\circ} \mathrm{E}$, alt. $1100 \mathrm{~m}$ ). It is shown how the. BRDF depends on the measurement geometry - incident and scatter angles and on the sample particle sizes. As a demonstration of the application of the results, airborne BRDF measurements acquired with NASA's Cloud Absorption Radiometer (CAR) over the same general site where the regolith samples were collected are compared with the laboratory results. Good agreement between laboratory measured and field measured BRDF is reported.
\end{abstract}

BRDF, Optical instrumentation and measurements, Metrology, Remote sensing

\section{INTRODUCTION}

The monitoring of land surface is a major science objective in Earth remote sensing. An important goal is to identify major biomes and to map and distinguish the changes in their composition introduced by anthropogenic and climatic factors. Currently, deforestation and desertification are the most important land cover area processes of scientific interest. A feasible way of characterizing surface anisotropy is by measuring the Bi-directional Reflectance Distribution Function (BRDF) for different surfaces and ecosystems in many parts of the world. The BRDF describes the spatial and spectral interaction of light on a sample's surface as a function of the incident and scatter angles and wavelength. BRDF measurements of Earth scenes can be used as a sensitive tool for early detection of changes occurring in vegetation canopies, soils, and oceans [1]. In this study, we analyzed laboratorybased BRDF data of soil samples collected at Etosha Pan, Fig. 1, which is situated in northern Namibia with an area of 4590 square kilometers. The Etosha Pan is a core site for validation of the Earth Observing System (EOS) Terra and Aqua satellite instruments. The pan is desert-like, white in color, and a dry salt pan without any vegetation. However, during the rainy years, Etosha pan becomes a $10 \mathrm{~cm}$ deep lake and a breeding ground for thousands of flamingos. The Etosha Pan mineralogy is dominated by four compounds: feldspar and mica, feldspar and sepiolite, silicates, and calcite and dolomite; compounds that determine the pan's spectra. Etosha pan surroundings are dominated by mopane and acacia trees and grasslands. All the samples were measured using NASA Goddard Space Flight Center's (GSFC's) Diffuser Calibration Laboratory (DCL) scatterometer [2]. The typical measurement uncertainty was $1 \%(k=1)$ or better, where $k$ is the coverage factor. The laboratory results were compared to BRDF measurements with an airborne radiometer, the Cloud Absorption Radiometer (CAR), which was developed at GSFC [3].

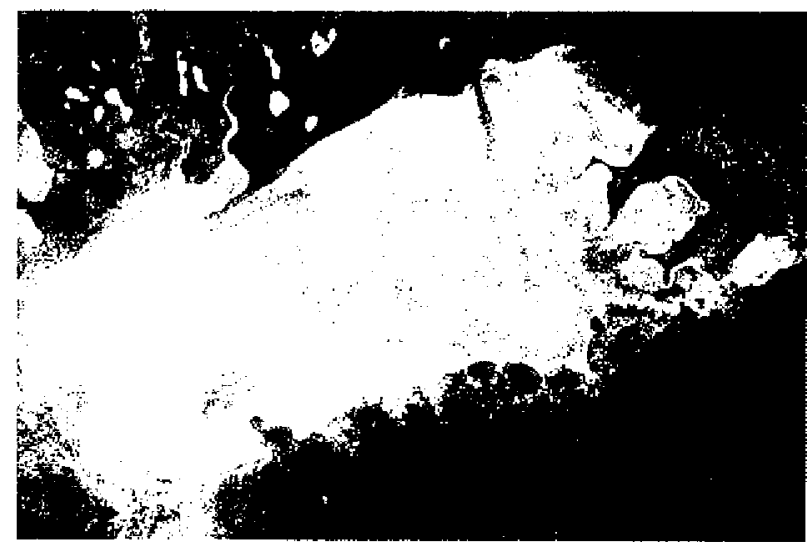

Figure 1. Etosha Pan

\section{METHODOLOGY}

The BRDF is a function which defines the spectral and spatial reflection characteristic of a surface. The definition and derivation of BRDF are credited to Nicodemus [4] who presented a unified approach to the specification of reflectance in terms of both incident and reflected light beam geometries for characterizing both diffuse and specular reflecting surfaces of optical materials. He defined the BRDF as a distribution function relating the irradiance incident from one given direction to the reflected radiance in another direction. Thus, the BRDF is presented in radiometric terms as the ratio of the radiance, $L_{r}$, reflected by a surface into the direction $\left(\theta_{r}, \phi_{r}\right)$ to the incident irradiance, $E_{i}$ on a unit surface area from a specified direction $\left(\theta_{i}, \phi_{i}\right)$ at a particular wavelength, $\lambda$. This is expressed mathematically as:

$$
B R D F=\frac{d L_{r}\left(\theta_{i}, \phi_{i}, \theta_{r}, \phi_{r} ; E_{i}\right)}{d E_{i}\left(\theta_{i}, \phi_{i}\right)},
$$

where the subscripts $i$ and $r$ denote incident and reflected light respectively, $\theta$ is the zenith angle, and $\phi$ is the azimuthal angle. 
Nicodemus further assumed that the incident beam has uniform cross section; the illumination on the sample is isotropic; and all scattering comes from the sample surface and none from the bulk. In practice, we are dealing with real samples' surfaces which are not isotropic and the optical beams used to measure the reflectance are not perfectly uniform. Hence from practical considerations the BRDF can be defined as presented by Stover [5] as the scattered power per unit solid angle normalized by the incident power and the cosine of the detector zenith angle. It is expressed in terms of incident power, scattered power, and the geometry of incident and reflected light:

$$
B R D F=\frac{P_{s} / \Omega}{P_{i} \cos \theta_{s}},
$$

where $P_{\mathrm{i}}$ is the incident power, $P_{\mathrm{s}}$ is the scatter power, $\theta_{\mathrm{s}}$ is the detector zenith angle, and $\Omega$ is the solid angle determined by the area of detector aperture, $A$, and the distance from the sample surface to the limiting aperture at the detector assembly, $R$. The solid angle can be computed as $\Omega=A / R^{2} . P_{\mathrm{i}}$ is the incident radiant power, and $\theta_{\mathrm{r}}$ is the reflected zenith angle. The $\cos \theta_{s}$ factor is a correction to account for the illuminated area, when viewed from the detector direction. BRDF has units of inverse steradians and can range from very small numbers (e.g. for off-specular black samples) to very large values (e.g. for highly reflective samples at specular reflectance). The BRDF angular convention is shown in Fig.2, where $\theta_{\mathrm{i}}$ is the incident zenith angle measured from the sample normal; $\phi_{i}$ is incident azimuth angle; $\theta_{\mathrm{s}}$ and $\phi_{\mathrm{s}}$ are scatter zenith and scatter azimuth angles, respectively.

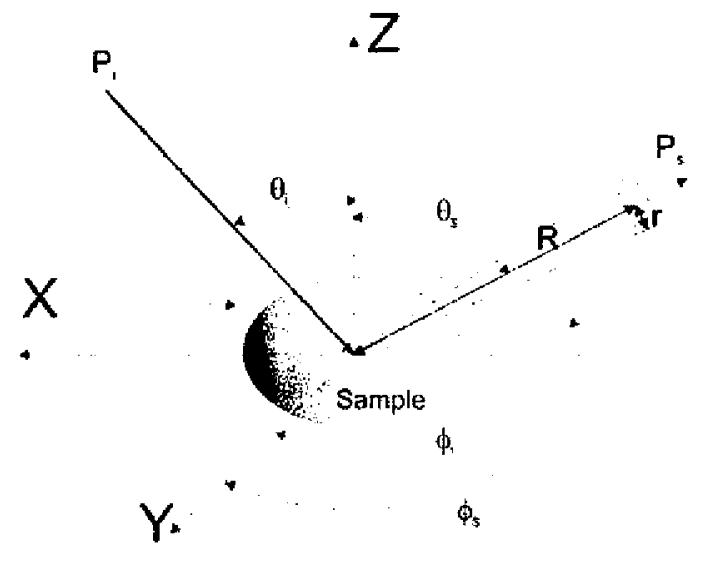

Figure 2. BRDF angular convention after Nicodemus

In the case of CAR measurements, the spectral BRDF $\left(\mathbf{R}_{\lambda}\right)$ is expressed following the van de Hulst [6] formulation, Fig. $3:$

$$
R_{\lambda}\left(\theta, \theta_{0}, \Phi\right)=\frac{\pi I_{\lambda}\left(\theta, \theta_{0}, \Phi\right)}{\mu_{0} F_{\lambda}},
$$

where $I_{\lambda}$ is the measured reflected intensity (radiance); $F_{\lambda}$ is the solar flux density (irradiance) incident on the top of the atmosphere; $\theta$ and $\theta_{0}$ are respectively the viewing and incident zenith angles; $\Phi$ is the azimuthal angle between the viewing and incident light directions; and $\mu_{0}=\cos \theta_{0}$. The $\mathrm{R}_{\mathrm{h}}$ is equivalent to the bidirectional reflectance factor (BRF) as defined by Nicodemus, which is dimensionless and numerically equivalent to BRDF times $\pi$.

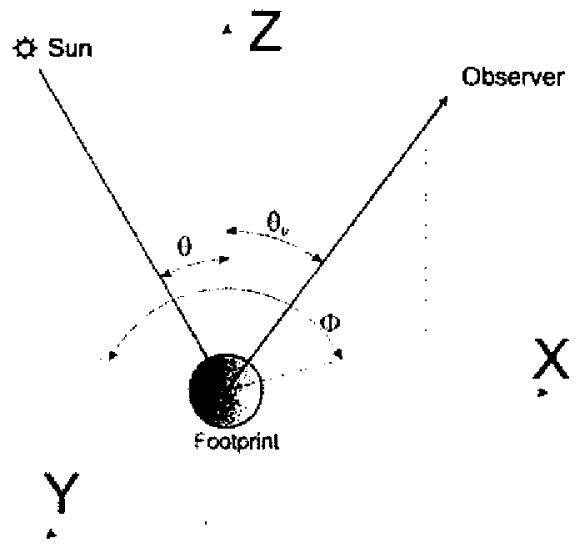

Figure 3. BRF angular convention after van de Hulst

The CAR instrument was flown aboard the University of Washington Convair CV-580 research aircraft during the Southern Africa Regional Science Initiative 2000 (SAFARI 2000) dry season campaign [7]. The CAR scan mirror scans $360^{\circ}$ in a plane perpendicular to the direction of flight with a rate of 100 scans per minute and the data is collected through a $190^{\circ}$ aperture. The analog signal is digitized internally to 16 bits. The CAR has an instantaneous field of view of $1^{\circ}$ and 14 channels located between 335 and $2344 \mathrm{~nm}$.

\section{EXPERIMENTAL}

\section{A. Measurement setup and samples}

In this study, we present laboratory BRDF measurements of regolith samples. in an effort to show the role of spatial and spectral vatiability of the natural biome, a continuation from our previous work [8]. The BRDF was measured using the scatterometer located in the Diffuser Calibration Laboratory at NASA's Goddard Space Flight Center (GSFC). The scatterometer, Fig.4 is located in a class 10000 laminar flow cleanroom.

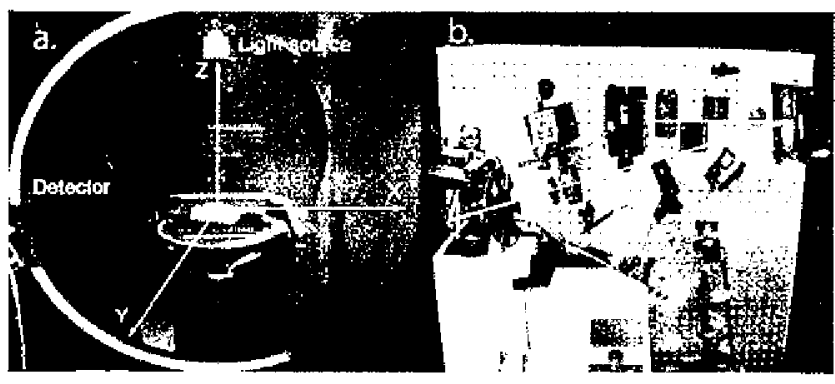

Figure 4. The Scatterometer: a. Goniometer with the "Rock" sample, b. Optical table

The scatterometer is capable of measuring the BRDF and BTDF of a wide range of samples including white and gray- 
scale diffusers, black painted or anodized diffusers, polished or roughened metal surfaces, clean or contaminated mirrors, transmissive diffusers, liquids, and granular solids. The operational spectral range of the instrument is from 230 to 900 nm. The scatterometer can perform principal plane and out-of principal plane BRDF measurements. It consists of a vertical optical source table, a sample stage, a detector goniometer, and a computer system for positioning control, data collection and analysis. The light source used was a short-arc Xenon lamp monochromator assembly producing an incoherent, tunable light with a well-defined spectral bandpass width. The samples were mounted horizontally on the sample stage and aligned with the scatterometer axes of rotation. The sample stage can be moved in the $X, Y$ and $Z$ linear directions using three motor stages. There is also an additional degree of freedom allowing sample rotation in the horizontal plane. The detector field-ofview was centered on each sample target for all measurements and was underfilled by the incident beam, meaning the illuminated area on the sample was always smaller than the detector FOV. Scattered light was detected using a polarization insensitive uitraviolet enhanced silicon photodiode with output fed to a computer-controlled lock-in amplifier. The detector assembly can be rotated around the vertical and horizontal axes of the goniometer. The surface of the sample to be measured was positioned at the cross point of the two perpendicular goniometer rotation axes which define the center of rotation of the goniometer system. All measurements were made for polarizations of the illumination beam both parallel, $-p$, and perpendicular, $-\mathrm{s}$, to the plane of incidence. The BRDF for each polarization was calculated by dividing the net signal from the transmitted radiant flux by the incident flux and the projected solid angle from the calibration item to the limiting aperture of the detector. This setup facilitates the acquisition of computerized BRDF measurements at different incident and scattered geometries for a complete data acquisition at selected points and wavelengths.

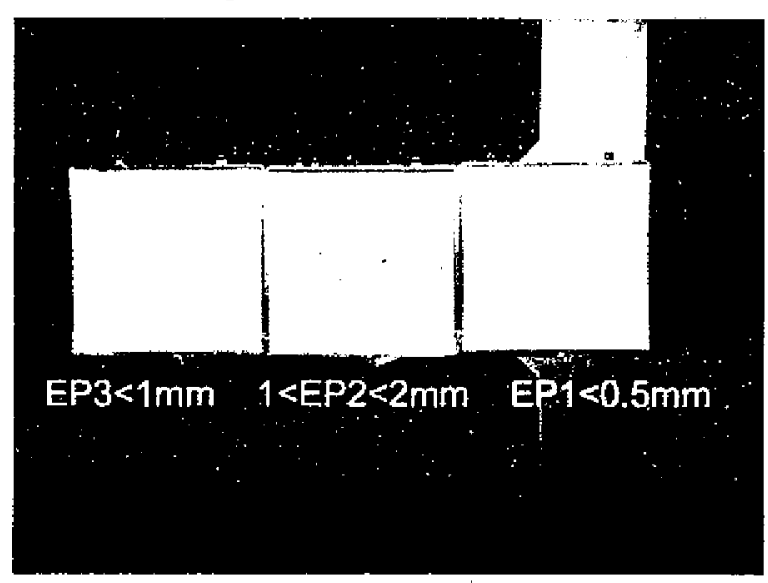

Figure 5. The regolith samples

Four fractions of the same sample material were studied, Fig.5. The first sample, "the rock", is a piece of rock sediment, hereafter referred to as EPR (Etosha Pan Rock), while the other three are regolith samples derived by grinding the sample material to different particle sizes. Particle sizes for the EP1 sample (Etosha Pan 1) was sub $0.5 \mathrm{~mm}$, for the EP2 sample was between 1 and $2 \mathrm{~mm}$, and for the EP3 sample was sub 1 $\mathrm{mm}$. Etosha Pan samples were studied at different wavelengths and incident and scatter angles corresponding to the airborne measurements over the Etosha Pan. The laboratory-based BRDF of all samples was analyzed in the principal plane at $412,555,667$ and $869 \mathrm{~nm}$, however; only $667 \mathrm{~nm}$ and $869 \mathrm{~nm}$ are similar to CAR's wavelengths. The samples were characterized at an incident angle of $30^{\circ}$ and at scatter zenith angles from $0^{\circ}$ to $80^{\circ}$ in steps of $5^{\circ}$. The scatter azimuth angles of $0^{\circ}$ and $180^{\circ}$ correspond to the principal plane measurement geometry. The samples were placed in square $50 \times 50 \times 5 \mathrm{~mm}$ black plastic holders with the sample surface well flattened and with uniformly sized particles distributed through the entire surface area.

\section{B. Results and Discussion}

The laboratory-based BRDF at $30^{\circ}$ incidence for the four Etosha Pan samples is shown in Fig. 6 at $667 \mathrm{~nm}$. The rock sample's BRDF is higher as the particulate incident light shadowing and scatter light obscuration effects are the smallest. The finest structure sample, EP1, has distinctively higher BRDF than the two other larger fractions, samples EP2 and EP3. It is worth noting that the shape of the BRDF curve for the rock sample is different than the shape of the particulate samples. It is also very important that all samples have apparent backscattering properties. Although the BRDF at $\theta_{\mathrm{i}}=\theta_{\mathrm{s}}$ could not be measured due to the relative geometries of the scatterometer source optics and detector, the BRDF for all samples shows evidence of a significant opposition effect represented by increased light being retroscattered back in the direction of the incident beam. Sample No.2, with particle sizes between 1 and $2 \mathrm{~mm}$, has the lowest BRDF.

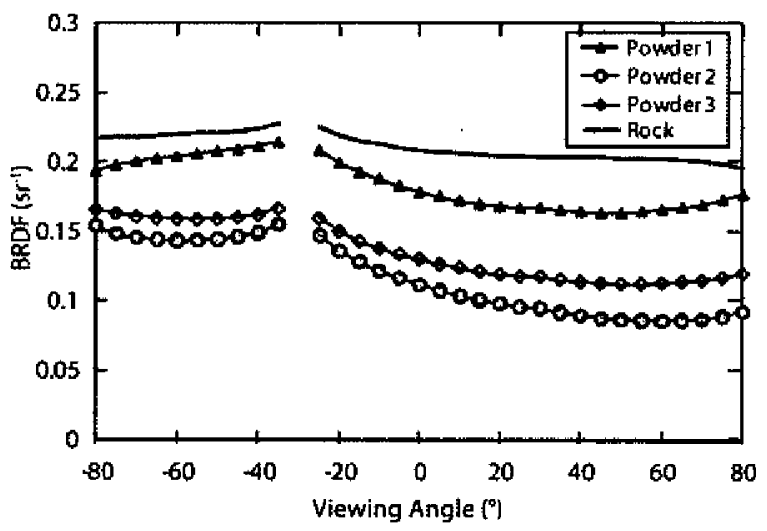

Figure 6. $\mathrm{BRDF}$ at $30^{\circ}$ incident angle and $667 \mathrm{~nm}$

In addition to BRDF measurements, the samples' spectral reflectance was measured with an Analytical Spectral Device (ASD) spectroradiometer in-plane at $30^{\circ}$ incident angle and $30^{\circ}$ scatter angle from 350 to $2500 \mathrm{~nm}$. This is shown in Fig. 7. The ASD spectra present a full reflectance picture for the 
VIS-NIR spectral range providing additional information on the Etosha Pan sample's reflectance properties.

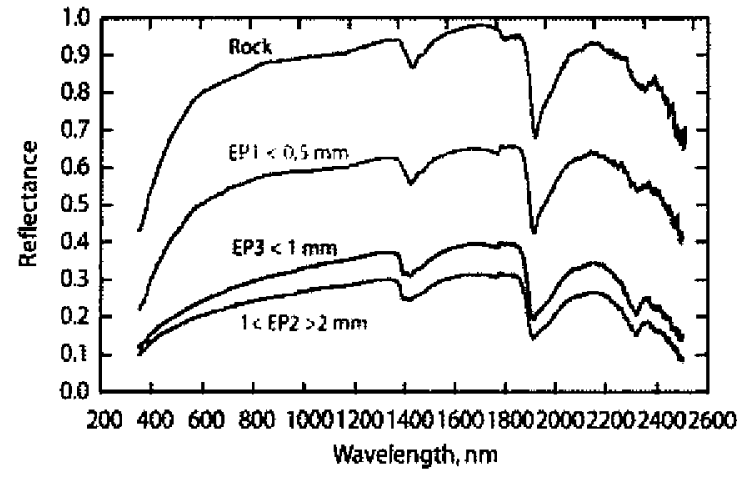

Figure 7. ASD spectra at $30^{\circ}$ incident angle and $30^{\circ}$ scatter zenith angle

In order to correctly compare the laboratory-based BRDF with the airborne measurements, we calculated the composite laboratory-based BRDF from the BRDF of the rock and 3 particulate Etosha Pan samples as measured in the laboratory. The contribution of each sample to the calculated composite laboratory-based BRDF was determined by the distribution of the 3 components as seen by the CAR instrument during airbome measurements. The components' spectral reflectance weighting was calculated by the field campaign participants after a careful examination of photographs of the flight track over Etosha Pan. These weights were determined to be $25 \%$ $\mathrm{EP} 1,50 \% \mathrm{EP} 2$, and $25 \% \mathrm{EP} 3$. The simulated fractional laboratory-based data is compared to the CAR airborne data.

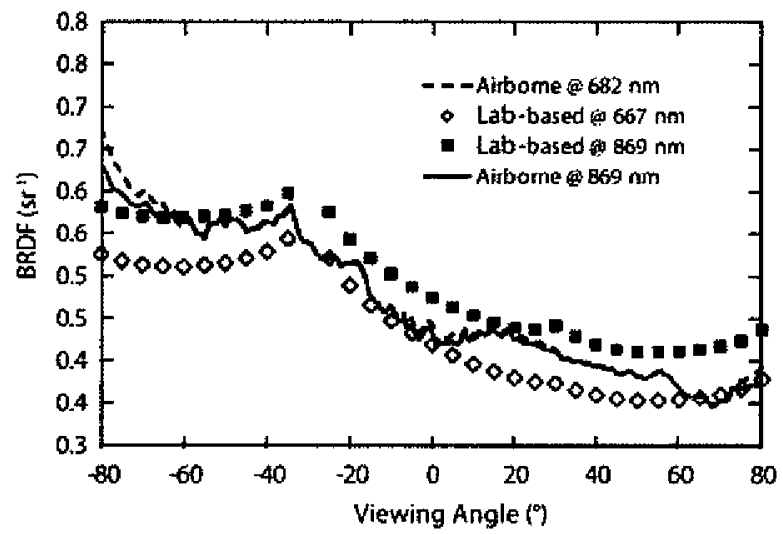

Figure 8. Etosha Pan simulated scene BRDF from the laboratory-based BRDF and CAR airbome data

The same general shape of the laboratory-measured samples and airborne measurements can be seen in Fig. 8. The data matches well within the uncertainty for both wavelengths over the scatter angle range with the exception of $-80^{\circ}$ where the CAR measured data are higher. However, the airborne data at the two wavelengths shown are very close. The laboratory based data show a larger difference between the data at 667 $\mathrm{nm}$ and $869 \mathrm{~nm}$.

\section{CONCLUSIONS}

This work is intended to describe more completely the BRDF of regolith samples from Etosha Pan measured in a laboratory environment. In addition, the laboratory results are compared to airborne measurements of these areas by the CAR instrument. In the laboratory measurements, the BRDF depends on the incident and viewing angles, on the nature of the sample, on the sample status, and on the particle size fraction. It is highest for the rock sample and lowest for the larger size particles regolith sample.

Laboratory-based and CAR airborne data sets from Etosha Pan were compared at 682 and $870 \mathrm{~nm}$ for the airborne data and 677 and $869 \mathrm{~nm}$ for the laboratory data, respectively. The BRDF curves have the same general shape, and the data matches well into the uncertainty for both wavelengths over all viewing angular range. However, the airborne data show smaller BRDF differences between the two wavelengths than the laboratory-based data. The effects of atmospheric absorption and scattering from CAR measurements could be a source of uncertainty. We believe the laboratory results are going to be of great use to the remote sensing community in their modeling and correction efforts of airborne data.

The results presented here are traceable to the National Institute of Standards and Technology's (NIST's) Spectral Trifunction Automated Reference Reflectometer (STARR).

\section{REFERENCES}

[1] J.J. Butler, B.C. Johnson, R.A. Bames, "The calibration and characterization of Earth remote sensing and environmental monitoring instruments", in Optical Radiometry, Eds. A.C. Parr, R.U. Datla, J.L. Gardner, Academic Press, New York, 2005

[2] G.T. Georgiev, J.J. Butler, "Laboratory-based bidirectional distribution functions of radiometric tarps", Applied Optics, vol.47, pp. 3313-3323, 2008

[3] M.D. King, M.G. Strange, P. Leone, L.R. Blaine, "Multiwavelength scanning radiometer for airbome measurements of scattered radiation within clouds", J. Atm, and Oceanic Techn., vol. 3, pp. 513-522, 1986

[4] F.E. Nicodemus, J.C. Richmond, J.J. Hsia, I.W. Ginsburg, T. Limperis, "Geometrical considerations and nomenclature for reflectance", National Bureau of Standards, NBS monograph 160, Oct. 1977

[5] J.C. Stover, "Optical scattering: measurement and analysis", SPIE Press, Bellingham, Washington, 1995

[6] H.C. van de Hulst, Multiple light scattering, Tables, Formulas, and Applications, vol. 1. San Diego: Academic Press, 1980.

[7] C.K. Gatebe, M.D. King, S. Platnick, G.T. Arnold, E.F. Vermote, B. Schmid, "Airborne spectral measurements of surface-atmosphere anisotropy for several surfaces and ecosystems over southem Africa", J. Geophysical Research, vol. 108, pp. 1-16, 2003

[8] G.T. Gcorgiev, C.K. Gatebe, J.J. Butler, M.D. King, "BRDF calibration of natural samples in support of remote sensing", IGARSS '07, pp.2877$2888,2007$. 